\title{
Generalized Multiple Equilibrium Paths for a Truncated Polyhedral Tensegrity
}

\author{
Seunghye Lee ${ }^{1}$, Quang Truong Phung ${ }^{1}$, and Jaehong Lee ${ }^{1}$
}

\begin{abstract}
Generalized form-finding procedure for a truncated polyhedral tensegrity is presented by using force density method combined with a genetic algorithm. A constrained minimization problem consisting of eigenvalues of the force density matrix and the standard deviation of the force densities of the cables is performed. Multiple force density curves are obtained and compared with those of previous investigations for a truncated icosahedral tensegrity in terms geometry, energy and total length ratio of cableto-strut. Various new shapes of tensegrity are obtained from present analysis by searching feasible self-equilibrium stable configuration of the structure.
\end{abstract}

Keywords - Tensegrity structures, Genetic algorithm, Formfinding, Truncated polyhedral tensegrity

\section{INTRODUCTION}

A tensegrity structure consists of a set of discontinuous compression components inside continuous tensile components [1]. Self-equilibrium analysis and stability properties are the fundamental problem in designing a tensegrity structure [2]. The design of tensegrity structures is divided into three steps: form-finding, structural stability and load analysis [3]. A key step in the design of tensegrity structures is a form-finding [4]. The form-finding method of tensegrity systems is a process of finding an equilibrium configuration. Over the past few years, several studies have been made on form-finding methods of the tensegrity systems.

Most of the literature reports how form-finding is influenced by initial topology (node connectivity) and initial force densities. These initial values tend to dominate final stability properties. Therefore, the present study is inspired by this fact: a genetic algorithm is applied to the process of obtaining initial force densities. Using direct encoding to obtain the initial force density values, it has been shown that this new algorithm could successfully demonstrate the formfinding process of any tensegrity structure. While performing a numerical example, this study found that the truncated regular tensegrity example give rise to new unknown configurations.

The present study explores most generalized form-finding results of a truncated polyhedral tensegrity using force density method combined with a genetic algorithm. The various families of the truncated polyhedral tensegrity including Zbased pattern as most investigators suggested, are obtained. \footnotetext{
Korea
}

Numerical result is presented for a truncated icosahedral tensegrity addressing the accuracy and robustness of the proposed method.

\section{II.EQUILIBRIUM AND STABILITY}

The force density method uses a linear equation in the nodal coordinates; this equation can be linearized using Eq. (1), known as force density.

$$
q_{k}=\frac{f_{k}}{l_{k}}
$$

where any member $\mathrm{k}$ has a member force $f_{k}$ and a length of element $l_{k}(k=1,2,3, \ldots, b)$. For a $d$-dimensional $(d=2$ or 3$)$ tensegrity structure with $b$ members and $n$ free nodes can be expressed by a connectivity matrix $\mathbf{C}$ as discussed in [5].

Let $\mathbf{x}, \mathbf{y}, \mathbf{z}$ denote the nodal coordinate vectors of the free node, in $x, y$ and $z$ directions. In a state of self-stress, external forces are considered to be zero and self-weights are ignored. As a result, the equilibrium equations can be written as follows:

$$
\mathbf{D}[\mathbf{x y z}]=\mathbf{C}^{T} \operatorname{diag}(\mathbf{q}) \mathbf{C}[\mathbf{x y z}]=[\mathbf{0 0 0}]
$$

where $\mathbf{D}$ is the force density matrix [6]. The force density matrix $\mathbf{D}$ is always square and symmetric [7] and semidefinite due to the existence of compression members (struts), with $q_{k}<0$. Using a second term of Eq. (2), the equilibrium equation can be expressed as.

$$
\begin{aligned}
& \mathbf{C}^{T} \operatorname{diag}(\mathbf{q}) \mathbf{C x}=[\mathbf{0 0 0}] \\
& \mathbf{C}^{T} \operatorname{diag}(\mathbf{q}) \mathbf{C y}=[\mathbf{0 0 0}] \\
& \mathbf{C}^{T} \operatorname{diag}(\mathbf{q}) \mathbf{C z}=[\mathbf{0 0 0}]
\end{aligned}
$$

Eq. (3) can be reorganized as

$$
\mathbf{A q}=0
$$

where $\mathbf{A}$ is known as the equilibrium matrix, defined by

$$
\mathbf{A}=\left(\begin{array}{l}
\mathbf{C}^{T} \operatorname{diag}(\mathbf{C x}) \\
\mathbf{C}^{T} \operatorname{diag}(\mathbf{C y}) \\
\mathbf{C}^{T} \operatorname{diag}(\mathbf{C z})
\end{array}\right)
$$

Eq. (2) shows the relationship between force density matrix D and nodal coordinates, and Eq. (4) illustrates the relationship between the equilibrium matrix $\mathbf{A}$ and force densities. 
The form-finding procedure for tensegrity structures requires rank deficiency conditions of force density and equilibrium matrices. The rank deficiency of $\mathbf{D}\left(n_{D}\right)$ has at least one state of self-stress, since the sum of the elements of the row or column of force density matrix $\mathbf{D}$ always equals zero. In a $d$-dimensional tensegrity structure, the rank deficiency of $\mathbf{D}$ has at least $d$ useful particular solutions. Therefore, the rank deficiency condition is defined as

$$
n_{\mathbf{D}}=n-r_{\mathbf{D}} \geq d+1
$$

where $r_{\mathbf{D}}=\operatorname{rank}(\mathbf{D})$.

The second rank deficiency condition is related to a dimension of null space of the equilibrium matrix A. The dimension of null space of the equilibrium matrix $\mathrm{A}$ is identical to "s", known as the number of independent states of self-stress. A tensegrity structure ensures the existence of at least one state of self-stress and can be stated as

$$
s=n_{\mathrm{A}}=b-r_{\mathbf{A}} \geq 1
$$

where $r_{\mathbf{A}}=\operatorname{rank}(\mathbf{A})$.

\section{FORM-FINDING PROCESS}

In the proposed method, the dimensions of the structure, the nodal connectivity and the type of each member are only required for a form-finding procedure. Firstly, the force density matrix is calculated from the initial force density vector. A genetic algorithm is then used to obtain the initial force density values that leads to the force density matrix to satisfy Eq. (2). The fitness function consists of two variable, $\alpha$ and $\beta$.

$$
\begin{aligned}
& \text { Minimize : } \alpha \beta \\
& \text { Subject to: }\left(\begin{array}{rr}
0<q_{i}<1 & \left(q_{i} \in \Gamma_{c}\right) \\
-1<q_{j}<0 & \left(q_{j} \in \Gamma_{s}\right)
\end{array}\right)
\end{aligned}
$$

in which

$$
\begin{gathered}
\alpha=\sum_{i=1}^{s}\left|\lambda_{i}\right| \\
\beta=\sqrt{\frac{\sum_{i=1}^{m} q_{i}^{2}}{m}-\left(\frac{\sum_{i=1}^{m} q_{i}}{n}\right)^{2}}
\end{gathered}
$$

and $\Gamma$ denotes the total set of the force density; $\Gamma_{\mathrm{c}}$ is the set of the force density for cable members and $\Gamma_{\mathrm{s}}$ is the set of the force density for strut members. Subscript ( $i$ and $j$ ) and superscript $(k)$ denote element numbers and group numbers, respectively. Eq. (9) indicates unilateral conditions for tensegrity grid structures, which is necessary to have a unique value of initial self-stress forces. In Eq. (8), $\alpha$ is the sum of the first $d+1$ smallest eigenvalues of the force density matrix as suggested by Koohestani [8]. The square force density matrix D can be factorized as follows by using the eigenvalue decomposition [9].

$$
\mathbf{D}=\boldsymbol{\Phi} \Lambda \boldsymbol{\Phi}^{T}
$$

where $\boldsymbol{\Phi}$ is the orthogonal matrix whose ith column is the eigenvector basis of $\mathbf{D}$. $\lambda$ is the diagonal matrix whose diagonal elements are the corresponding eigenvalues, i.e., $\Lambda_{i i}=\Lambda_{i}$. The eigenvector of $\boldsymbol{\Phi}$ corresponds to eigenvalue $\lambda_{i}$ of $\Lambda$. The eigenvalues are in increasing order as

$$
\lambda_{1} \leq \lambda_{2} \leq \cdots \leq \lambda_{n}
$$

In Eq. (8), $\beta$ is the standard deviation of the force density in the cables. The role of $\beta$ is to make uniform the force density values so that the value of the fitness function can be minimized. The notation $m$ is the number of cables. Koohestani [8] also presented a variable that significantly improves the behavior of fitness function. However, in this paper, the more effective variable is used to not only significantly improve the behavior of the fitness function but also provide uniformity to the force density values.

It is clear that the number of zero eigenvalues of $\mathbf{D}$ is equal to the dimension of its null space. The first $d+1$ eigenvectors of $\boldsymbol{\Phi}$, corresponding to the first $d+1$ smallest eigenvalues, respectively, are chosen as nodal coordinates $[\mathbf{x}, \mathbf{y}, \mathbf{z}]$ for a $d$ dimensional tensegrity structure.

\section{TRUnCATED PolyhedRAL TENSEGRITY}

The Overall, there are five convex regular polyhedra, including tetrahedron, cube, octahedron, dodecahedron and icosahedron. By truncating uniformly each vertex of these regular polyhedra, the truncated regular polyhedra can be obtained [10]. Truncated forms of tetrahedron, dodecahedron and icosahedron are illustrated in Fig. 1. The tensegrity can be defined as two major classes, called Z-based structures and rhombic structures, respectively [1]. The Z-based tensegrity structure conforms to a rule of the Z-shape. In a Z-based truncated regular polyhedral tensegrity, some cables coincide with a cutting edge of the truncated polyhedron and the struts connect the vertexes by the rule of the Z-shape [11]. The rhombic tensegrity structure is composed of cells that consist of four cables around one strut, and the configuration in tensegrity structures of a Z-based cell and a rhombic cell are illustrated in Fig. 2 [12].

The truncated tetrahedral tensegrity is the simplest type of the truncated polyhedral tensegrities. An analytical solution for regular (Z-based) truncated tetrahedral tensegrity was proposed by several authors [4, 13]. Also, the initial shapes and pre-stress modes for regular icosahedral and dodecahedral tensegrity modules were found analytically by [14]. Many researchers have presented form-finding results for the force density of the tensegrities as compared with the corresponding analytical solutions. In this study, new formfinding results are presented targeting these three truncated polyhedral tensegrities.

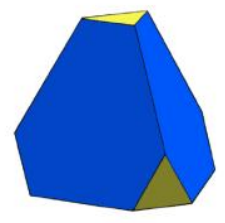

(a)

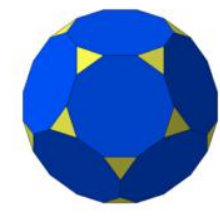

(b)

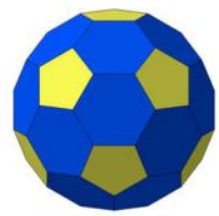

(c)
Fig. 1: Truncated regular polyhedral; (a) Truncated tetrahedron (b) Truncated dodecahedron, (c) Truncated icosahedron 


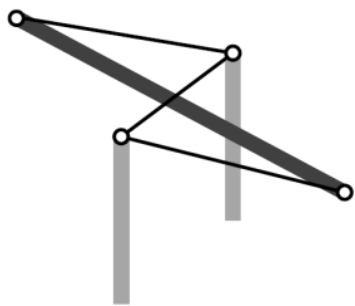

(a)

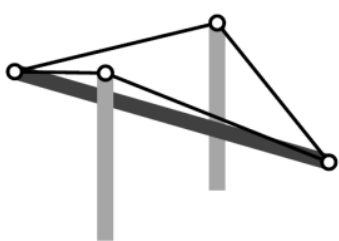

(b)
Fig. 2: Configuration in tensegrity structures of (a) a Z-based cell (b) a rhombic cell

\section{V.NUMERICAL EXAMPLES}

In this section, a numerical example of truncated icosahedral tensegrity is presented to demonstrate newly developed multiple equilibrium paths for force densities of the structure. Based on the algorithm developed, both the nodal coordinates and the single integral feasible force density vector are simultaneously defined with limited information of the nodal connectivity and the type of each member. The range of all the obtained graph limits its scope of the axis to 4.0 and 2.0 for the force densities of struts and cables, respectively. Each run of the genetic algorithm is conducted for 100 maximum generations, using a population size of 100. The tolerance in Eq. (9b) is set to 0.01 for all the analysis.

\subsection{Truncated Icosahedral Tensegrity}

The regular truncated icosahedral tensegrity has 60 nodes and 120 elements that consist of 30 identical compression members and 90 identical tension cables. After the proposed form-finding procedure, all results formed to have one selfstress state $(\mathrm{s}=1)$ and 55 infinitesimal mechanism modes. qt, qv and qb are denoted by the force densities in the truncatingedge cables, vertical cables and struts, respectively [15].

As shown in Fig. 3, four different results of the truncated icosahedral tensegrity in self-equilibrium are obtained. The four lines refer to a comparison between the results of qv versus $\mathrm{qb}$ where the truncated edge cables have typical force densities ( $q \mathrm{t}=1.0$ ). As expected, the force density curve of family 1 is in perfect agreement with the corresponding analytical solution [14]. Koohestani and Guest [16] also presented three sets of force densities and the final geometries of the three cases. These three sets of force densities are compared with present results in Fig. 3. Case 1 ( $q v=0.69, q b$ $=-0.33)$ lies in family 1 , while case $2(q v=0.77, q b=-0.53)$ and 3 ( $q v=7.21, q b=-1.25)$ lie in the equilibrium path of family 2. Case 3 is not marked in Fig. 3 because the range of force density is beyond the scope of the axis of Fig. 3 In this case, except for family 1 they do not also mention the typical lines of the various families. Fig. 4 shows four representative self-equilibrium configuration of truncated icosahedral tensegrities as all the cables with unit value $(\mathrm{qt}=\mathrm{qv}=1.0)$. The geometry of family 1 shows a form of typical regular truncated icosahedral tensegrity where the cables lie along the edges of the structure. Comparison of the total length ratio ( $\sum$ lc $/ \sum$ ls) and energy where all of the cable has typical force densities ( $\mathrm{qt}=\mathrm{qv}=1.0$ ) is presented in Table 1. Thus, the higher family number, the greater value of total length ratio and strain energy density is obtained. These results tend to be similar to those of truncated dodecahedral tensegrity. These results also show that a regular form is effective in terms of energy.

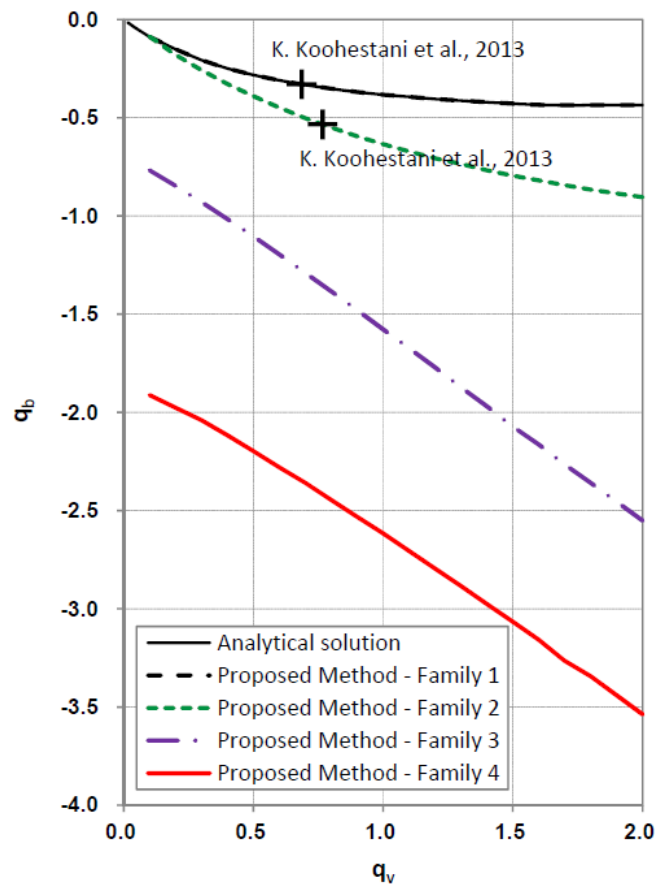

Fig. 3: Comparison between the results of $q_{v}$ versus $q_{b}$ for a truncated icosahedral tensegrity

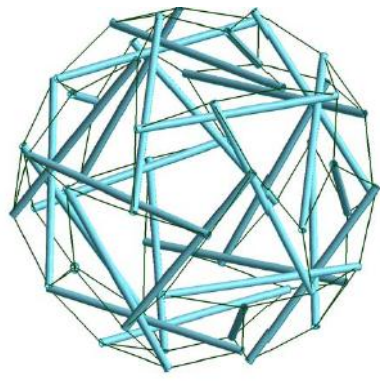

(a)

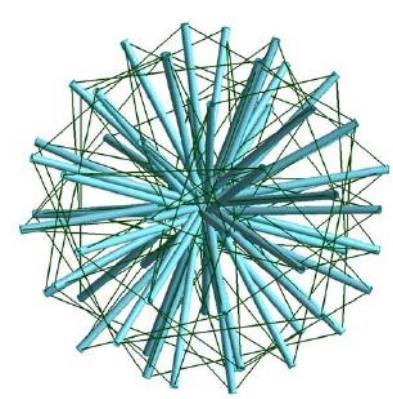

(c)

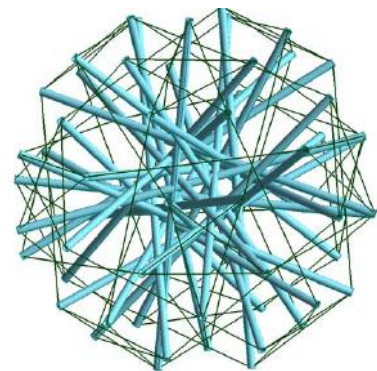

(b)

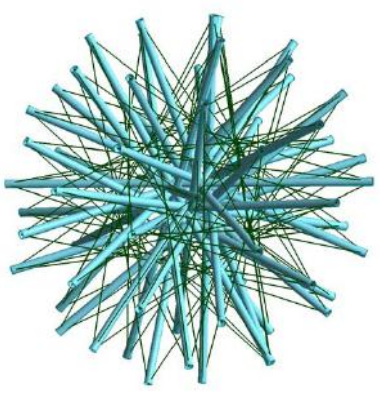

(d)
Fig. 4: Four representative self-equilibrium configuration of truncated icosahedral tensegrities: (a) Family 1 (b) Family 2 (c) Family 3 (d) Family 4 
TABLE I

Comparison Of The Total Length Ratio AND ENERgy In CASE That All Of THe CABle Has A Value OF 1.0 (TRUNCATED ICOSAHEDRAL TENSEGRITY)

\begin{tabular}{c|c|c|c|c}
\hline $\begin{array}{c}\text { Results for } q_{t} \\
=1.0\end{array}$ & $q_{v}$ & $q_{b}$ & $\sum l_{c} / \sum l_{s}$ & $\begin{array}{c}\text { Strain energy } \\
\text { density }\end{array}$ \\
\hline Family 1 & 1.0 & -0.4 & 1.1 & 1.2 \\
Family 2 & 1.0 & -0.6 & 1.3 & 5.9 \\
Family 3 & 1.0 & -1.6 & 2.1 & 24.4 \\
Family 4 & 1.0 & -2.6 & 2.8 & 56.0 \\
\hline \hline
\end{tabular}

\section{CONCLUSION}

In this study, a generalized form-finding method of the truncated polyhedral tensegrity is presented by using a force density method combined with a genetic algorithm. Multiple equilibrium paths of the force density for the truncated icosahedral tensegrity can be obtained. Based on the theoretical developments and numerical investigation, the following remarks can be made:

- While most of previous investigators presented Z-based geometry only for the truncated polyhedral tensegrities, various new families of the tensegrity shapes are generated by adjusting the range of the force density of the struts.

- Among all the families of the geometry for the truncated polyhedral tensegrity considered, Z-based geometry is formed to be most effective in terms of strain energy density and cable-to-strut length ratio.

- The nodal connectivity and unilateral conditions of the elements of the tensegrity do not change during the whole process of form-finding even for different families of force density curves.

As a natural extension of the present research, the various form-finding method for other types of tensegrities awaits further attention.

\section{ACKNOWLEDGMENT}

This research was supported by a grant (NRF2015R1C1A2A01055897) from NRF (National Research Foundation of Korea) funded by MEST (Ministry of Education and Science Technology) of Korean government.

\section{REFERENCES}

[1] Pugh, Anthony. An introduction to tensegrity. Univ. of California Press, 1976.

[2] Masic, Milenko, Robert E. Skelton, and Philip E. Gill. "Algebraic tensegrity form-finding." International Journal of Solids and Structures 42.16 (2005): 4833-4858.

http://dx.doi.org/10.1016/j.ijsolstr.2005.01.014

[3] Schenk, Mark. "Statically balanced tensegrity mechanisms." A literature review. Department of BioMechanical Engineering. Delft University of Technology (2005).

[4] Tibert, A. G., and Sergio Pellegrino. "Review of form-finding methods for tensegrity structures." International Journal of Space Structures 18.4 (2003): 209-223.

http://dx.doi.org/10.1260/026635103322987940

[5] Schek, H-J. "The force density method for form finding and computation of general networks." Computer methods in applied mechanics and engineering 3.1 (1974): 115-134.

http://dx.doi.org/10.1016/0045-7825(74)90045-0
[6] Estrada, G. Gomez, H-J. Bungartz, and Camilla Mohrdieck. "Numerical form-finding of tensegrity structures." International Journal of Solids and Structures 43.22 (2006): 6855-6868. http://dx.doi.org/10.1016/j.ijsolstr.2006.02.012

[7] Lee, Seunghye, and Jaehong Lee. "Form-finding of tensegrity structures with arbitrary strut and cable members." International Journal of Mechanical Sciences 85 (2014): 55-62. http://dx.doi.org/10.1016/j.ijmecsci.2014.04.027

[8] Koohestani, K. "Form-finding of tensegrity structures via genetic algorithm." International Journal of Solids and Structures 49.5 (2012): 739-747.

http://dx.doi.org/10.1016/j.ijsolstr.2011.11.015

[9] Meyer, Carl D. Matrix analysis and applied linear algebra. Siam, 2000. http://dx.doi.org/10.1137/1.9780898719512

[10] Zhang, Li-Yuan, et al. "A unified solution for self-equilibrium and superstability of rhombic truncated regular polyhedral tensegrities." International Journal of Solids and Structures 50.1 (2013): 234-245. http://dx.doi.org/10.1016/j.ijsolstr.2012.09.024

[11] $\mathrm{Li}$, Yue, et al. "Constructing tensegrity structures from one-bar elementary cells." Proceedings of the Royal Society A: Mathematical, Physical and Engineering Science 466.2113 (2010): 45-61. http://dx.doi.org/10.1098/rspa.2009.0260

[12] Feng, Xi-Qiao, et al. "Design methods of rhombic tensegrity structures." Acta Mechanica Sinica 26.4 (2010): 559-565. http://dx.doi.org/10.1007/s10409-010-0351-6

[13] Motro, Rene. Tensegrity: structural systems for the future. Elsevier, 2003. http://dx.doi.org/10.1016/b978-190399637-9/50038-x

[14] Murakami, Hidenori, and Yoshitaka Nishimura. "Static and dynamic characterization of regular truncated icosahedral and dodecahedral tensegrity modules." International Journal of Solids and Structures 38.50 (2001): 9359-9381. http://dx.doi.org/10.1016/S0020-7683(01)00030-0

[15] Linkwitz, Ing K., and H-J. Schek. "Einige bemerkungen zur berechnung von vorgespannten seilnetzkonstruktionen." Ingenieur-Archiv 40.3 (1971): 145-158. http://dx.doi.org/10.1007/BF00532146

[16] Koohestani, K., and S. D. Guest. "A new approach to the analytical and numerical form-finding of tensegrity structures." International Journal of Solids and Structures 50.19 (2013): 2995-3007. http://dx.doi.org/10.1016/j.ijsolstr.2013.05.014 